\title{
Perspectives on Aging
}

Demographic changes in the United States have long indicated that we are an aging society. The fastest growing segment of the older population are the centenarians, those over age 100, while the second fastest growing numbers of people are those over age 85 (Jacelon, 2018). Many gerontologists agree that it is necessary to differentiate between those who are "young-old" (65-74), those who are "middle-old" (75-84), and those who are "old-old" (85+).

Most service providers agree that because needs are generally different in young-old and old-old individuals, services and programs should be planned, oriented, and delivered in different ways for each group. In general, the young-old need more programs and services to reintegrate them into meaningful roles and activities after retirement, whereas the old-old tend to need supportive and protective programs and services. However, the aging process is so highly individualized that some young-old need supportive and protective services and other older adults prefer and need reintegrative programs and services. Individuals age biologically, psychologically, sociologically, and spiritually, as well as chronologically (Touhy, 2012). Chronological age is not an accurate predictor of physical condition or behavior, but it is used for convenience and for certain legal purposes (such as voting, Social Security eligibility, and so on). Using the distinctions "young-old," "middle-old," and "old-old" serves to focus attention on the enormous diversity of older adults and suggests differentiations must be made in this large segment of the population if we are to provide effectively for all its needs.

People become more unique as they grow older, not more alike. Because of this, and because aging is a distinct part of the life cycle not yet personally experienced by most of those who work with older adults, understanding older people is difficult for many in the helping professions. In our attempts to understand others, we often lean heavily on our own personal experiences and can therefore empathize reasonably well with a child, adolescent, or young adult. To understand the behavior and perspective of older adults, though, it is necessary to project ourselves into an age context with which we have no personal experience. This is not easy to do and is one of the challenges in working effectively with older adults.

The academic study of the aging process includes gerontology, the broad study of the aging process, and geriatrics, a specialty concentrating on medical problems associated with growing older. Gerontology incorporates multidisciplinary concepts and approaches in an attempt to understand all aspects of the complex aging process. Three academic areas have traditionally contributed substantially to gerontology: biological aging, concerned with longevity and how (and why) the body changes as aging occurs; psychological aging, concerned with adaptive capabilities, including memory, intelligence, and how individuals cope with their own aging; and social aging, concerned with social roles and expectations for older adults in a particular culture or society. 
However, many other academic disciplines also contribute to the study of aging because understanding aging is truly a multidisciplinary endeavor.

\section{BASIC CONCEPTS IN PHYSICAL AGING}

Research continues to indicate a much more optimistic picture of the aging process than previously presented. There are increased efforts now to differentiate "normal" aging from disease or pathology. It is clear that aging is not synonymous with illness or disease. True, certain aspects of the aging process make individuals more vulnerable to illness and disease, but no pathology is inevitable with age. Numerous physical changes historically attributed to aging are now recognized as more likely to be caused by lifestyle variables. For example, aches and pains traditionally attributed to aging more likely are due to a sedentary lifestyle or disuse of abilities rather than to aging per se. "Use it or lose it" is a common adage in gerontology and applies to physical, psychological, and social aging. Those skills and abilities that we continue to use will be maintained well into older age (barring accident or disease), whereas those we do not use will be lost.

Obviously, some factors associated with aging are nonmodifiable, such as genetics, gender, and age, but others can be modified by lifestyle, such as exercise, adequate nutrition, no smoking, and stress management. Because a substantial part of the aging process depends on lifestyle, we as individuals can make significant choices to increase the probability of healthy, positive aging. Three lifestyle factors that have a major impact on the manner in which we age are regular exercise, proper nutrition, and stress management. These and others will be discussed in this book.

\section{LESSENED RESERVE CAPACITY}

The major age-related change in the body is a lessened reserve capacity. All organ systems of the body have a substantial reserve capacity available to deal with high-demand or high-stress situations. With aging, there is a lessened reserve capacity in all the organ systems. Behavioral implications of lessened reserve capacity include the following:

1. Slowness. Although the aging process varies between one person and another, we all become slower with age. Most older adults are somewhat slower than they once were when taking in, processing, and acting on information. A fast-paced younger person will probably be a fast-paced older person but will be slower than when younger. Being slower in a fast-paced society is difficult, but it is important to realize that slowness is not synonymous with incompetence. Older adults who are allowed to pace themselves according to their own preferred schedule generally perform exceedingly well, whereas those who are forced into a schedule that is faster than they prefer are likely to perform much less well.

2. Stress. The body calls on its reserves to deal with high-stress or prolonged stress situations. The effect of stress tends to be greater on older adults because of their lessened reserve capacity. Being able to pace stressful situations appropriately helps older adults offset the effect of lessened reserve capacity.

3. Homeostatic equilibrium. Homeostatic equilibrium becomes more precarious as reserve capacity decreases with age. Homeostasis refers to a dynamic equilibrium that must be maintained in the body's internal environment. All the body's cells depend on a constant internal environment in order to function properly. Although a range of variation is possible in the internal environment, if homeostatic processes such as blood pressure, blood gases, acid-base balance (acidity or alkalinity of blood), and blood sugar become too high or too low, the individual will not survive. Highly complex regulatory mechanisms in the body help maintain homeostatic equilibrium, but with age and a lessened reserve capacity, it is easier 
for homeostatic balance to be disrupted, and once disrupted it is difficult to restore. For this reason, older adults are more vulnerable to illness, disease, and accidents. Biological aging is sometimes considered to be a decline in the ability to maintain homeostatic equilibrium, leading to impaired functioning and ultimately to death. It is therefore necessary for older adults to be particularly attentive to health maintenance behaviors and healthy lifestyles.

4. Pacing. Being able to pace oneself, or doing things in one's preferred way and time frame, becomes increasingly important in older age as one way to decrease the effect of lessened reserve capacity. Those who work with older adults need to allow for pacing if they wish to help them perform effectively and competently.

\section{PATTERNS OF DISEASE}

Illness and disease are not uncommon in older adults, although no specific disease is inevitable in older age. As researchers become more concerned with the dynamics of normal aging, and as health promotion and education for older adults become more available, many diseases currently associated with aging may be prevented, or at least delayed until extreme old age, by healthy lifestyle choices and greater attention to health maintenance.

Diagnosing and treating illness and disease in older adults become complex for the following reasons:

1. Many older persons have several health problems that need to be treated concurrently (comorbidity). A new problem may be masked by one already existing, and medications desirable for one health problem may exacerbate an existing health condition.

2. The symptoms older persons describe may not be the classic symptoms characteristic of younger individuals. For example, older adults may have a "silent" heart attack and not experience the classic or usual symptoms, or a ruptured appendix may be reported as an upset stomach or abdominal cramps.

3. Older adults tend to expect pain and discomfort as they age and may not report symptoms until a medical problem is far advanced. Those who work with older adults need to encourage them to report unusual symptoms that arise and not assume they are just signs of "old age."

Because greater numbers of people are living into older age than ever before, an accurate understanding of how body functions change with age and the implications of those changes is becoming increasingly important for all who work with older adults and for those who wish to know more about the best kind of preparation for their own old age.

What this book attempts to convey can be summarized in four major perspectives:

1. Recognition of aging as a highly diverse process. Chronological age is not a reliable predictor of specific organ system efficiency. There is enormous variation in the rate of the aging process both among individuals of the same chronological age and also in the body systems of a given individual. This is because some organ systems age more rapidly than others depending on heredity, diet, exercise, and stresses caused by past illnesses and the environment.

2. Importance of reserve capacity. In spite of individual variations, the body organ systems, unless stressed, generally continue to function quite adequately in older age, although there is some loss of reserve capacity. Stress results in reduced efficiency or inability to cope. Proper nutrition, exercise, pacing oneself, and regulating the environment to be maximally supportive are all positive ways to help offset the effect of physical aging on the body.

3. Accident prevention. Age-related physical changes increase the possibility of accidents and injury. Older persons and those working with them need to become extremely sensitive 
to and aware of situations that may contribute to accidents. Recovery time from accidental injury is usually longer for older adults, and often accidents are the first step in the transition from independence to dependence. Consequently, accidents have profound physical, psychological, and social significance for older adults' lives, and every effort should be directed toward preventing them.

4. Increased health promotion and health maintenance activities. Older adults are more susceptible to disease than the young. Physical changes associated with aging leading to loss of body reserves increase the older person's vulnerability to illness. Greater emphasis on health promotion, disease prevention, and health maintenance education for older adults allows them to become more actively involved in their own health and to feel they have more control over aging.

We do not intend, by the recitation of the physical changes and diseases associated with aging, to overemphasize decline and deterioration as an inevitable part of growing old. Indeed, many individuals are not drastically handicapped by age-related changes in their body systems. We believe others would be less impaired if they knew more about health promotion and health maintenance along with ways to adapt more efficiently to their individual aging process. The detrimental effects of age are a threat to self-image, to feelings of self-worth, and to independence, all of which are crucial to a satisfying and enjoyable life. Although physical changes are a reality of growing old, there are numerous ways to mitigate their effects and cope with them so as to at least partially offset the disabilities or limitations they impose. We suggest that gerontology and other helping professions place more research and educational efforts in helping older adults cope and adapt most effectively.

Although there are many different formulas for "successful aging," most include the following:

- Admit and accept the reality that aging imposes some limitations. Conserve energy; keep involved with life; make appropriate choices about use of time; and pace life realistically in accordance with needs, desires, and abilities.

- Be willing to change or modify lifestyle as necessary, especially physical activities and social roles. Remain flexible both mentally and emotionally. Reduce stress whenever possible. Plan a lifestyle to minimize disabilities and maximize remaining abilities.

- Develop new standards for self-evaluation and new goals. Measure self-worth by inner values such as the quality of human relationships, spirituality, appreciation of life, and not just by how much one can produce and achieve. Be a graceful receiver as well as a graceful giver. Older age can be a time of creativity and self-actualization if we choose to make it so.

\section{REFERENCES}

Jacelon, C. (2018). The aging population. In K. Mauk (Ed.), Gerontological nursing: Competencies for care (4th ed., pp. 23-40). Jones \& Bartlett.

Touhy, T. A. (2012). Gerontological nursing and an aging society. In T. A. Touhy \& K. Jett (Eds.), Toward healthy aging: Human needs and nursing response (8th ed., pp. 1-18). Elsevier Mosby. 